\title{
Topical Imiquimod Treatment of Lentigo Maligna
}

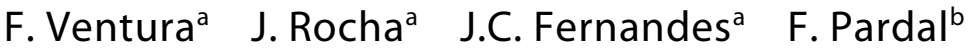 \\ C. Brito ${ }^{a}$
}

Departments of a Dermatology and Venereology and ${ }^{b}$ Pathology, Hospital de

São Marcos, Braga, Portugal

\section{Key Words}

Lentigo maligna $\cdot$ Lentigo maligna melanoma $\cdot$ Imiquimod

\begin{abstract}
Lentigo maligna (LM) is the in situ phase of lentigo maligna melanoma, which may progress to invasive melanoma if left untreated. It mainly occurs on sun-exposed areas of elderly patients. The lesions can be large and conventional surgery can be difficult, particularly on the face. Recent reports indicate that topical imiquimod $5 \%$ cream is effective in the treatment of LM. It may be an alternative when surgery or other classical treatments are not possible in elderly patients. We describe an 80-year-old Caucasian woman with a 10-year history of a histologically verified extensive LM of the face. She was treated with imiquimod $5 \%$ cream once daily. After four months it showed complete clinical response. One year after the treatment the patient was still free from recurrence.
\end{abstract}

\section{Introduction}

Lentigo maligna (LM), the in situ phase of lentigo maligna melanoma (LMM), may progress to invasive melanoma if not treated. It mainly occurs on chronically sun-exposed areas, typically the face, of middle-aged and elderly individuals. Surgical excision is the treatment of choice providing a cure in $90 \%$ of patients [1]. However this may be impractical or impossible due to cosmetic or functional impairment, comorbidity or patient preference. Nonsurgical treatments, such as cryotherapy and radiotherapy, are frequently used instead. More recently, imiquimod seems to be an interesting therapeutic alternative in the treatment of LM.

Imiquimod is a topical immunomodulator which can generate a local cytotoxic response with potentially antiviral and antitumor effects. It acts through the Toll-like receptors 7 and 8 and activation of the transcription factor NF-kB which is followed by the release of various Th1 cytokines [2]. Ahmed and Berth-Jones first reported the use of topical imiquimod in the treatment of LM [3]. Since then many cases of success have been reported $[1,4-7]$. A recent study comprising 48 patients with a longer described 
follow-up period (49 months on average), showed a LM clearance rate of 79\% [1]. In that series the response rate was lower than previously reported in the literature $[4,6]$.

Although rare, there are some cases of concern, such as an invasive melanoma that occurred in a patient after 1 month of imiquimod treatment [6] as well as the progression of LM lesions to amelanotic LMM following imiquimod therapy [8]. There are descriptions of a case of LM that almost cleared clinically but did not change histologically [9] and of the recurrence of LM 9 months after treatment [10].

\section{Case Report}

An 80-year-old Caucasian woman presented with a 10-year history of an extensive pigmented lesion of the face. Ten years before she was referred to our department with a pigmented macule on her left cheek. The skin biopsy showed typical features of LM. The patient refused surgical treatment and left the hospital. Eight years later she returned with serious complaints.

Physical examination revealed a brownish irregularly shaped macule, over the left hemiface, and two ulcerated nodules on the left cheek (fig. 1a). Dermatoscopic evaluation of the macule revealed a pseudo-network with asymmetrically pigmented follicular openings, rhomboidal structures and slate-grey pigmented areas. Excisional biopsy of the nodules was done and histopathological examination confirmed the evolution to melanoma with $7 \mathrm{~mm}$ of Breslow and Clark level IV. Three 4-mm punch biopsies taken from different areas of the macule revealed intraepidermal proliferation of atypical melanocytes, epidermal atrophy and solar elastosis consistent with the diagnosis of LM. The patient had no adenopathy or additional lesions on physical examination. Clinical staging, including hematologic examination and brain, chest, abdominal and pelvic CT scan were normal. Treatment with imiquimod 5\% cream was prescribed once daily. Four weeks later she developed an intense inflammatory response and the treatment was interrupted for one week (fig. 1b). After four months she showed complete clinical response (fig. 1c). A new 4-mm punch biopsy, three months after the treatment, showed no evidence of LM. One year after the treatment the patient was still free from recurrence.

\section{Discussion}

It is confirmed that imiquimod may play a role as an alternative in the management of LM. We found it the better choice for this elderly patient, especially given the extension of the lesion on the face. Furthermore she had refused the surgery and this was a palliative treatment. However there are several concerns about its use, particularly the lack of tissue for histological examination of margins, so additional studies are required. 
Fig. 1. Extensive LM of the face. a Before treatment with imiquimod. b Cutaneous inflammation after 1 month of treatment. c Clinical remission after 4 months of treatment.
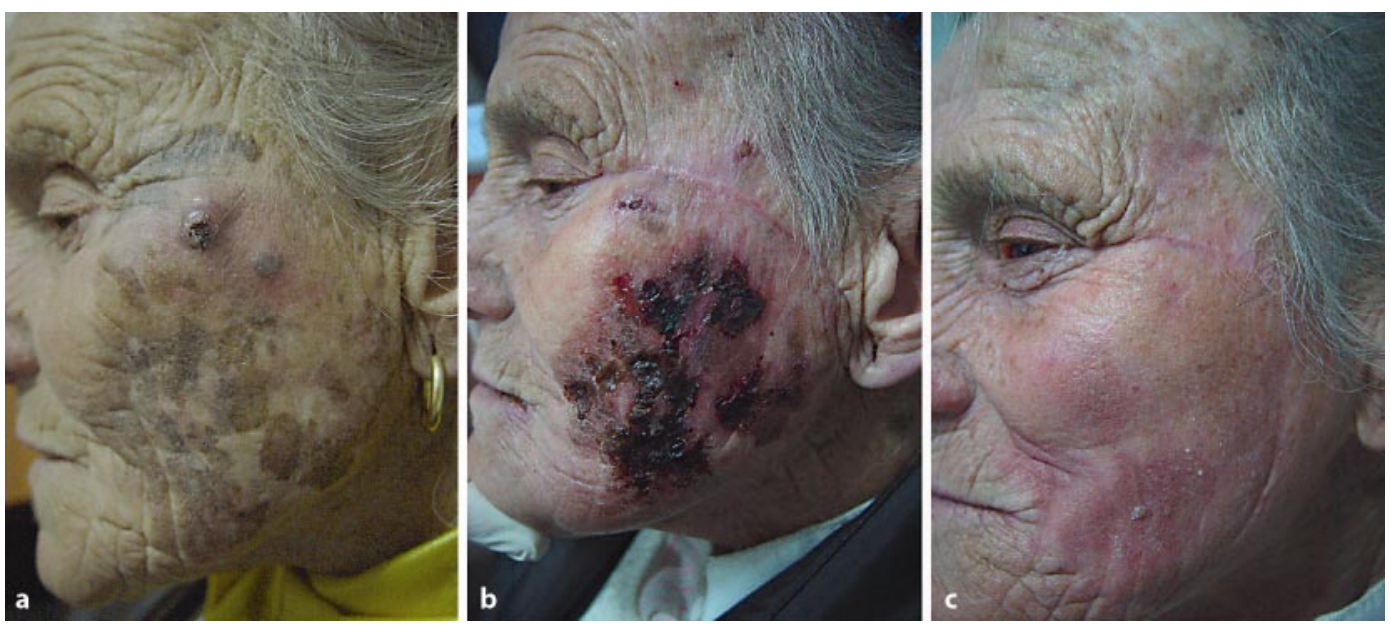


\section{References}

1 Powell AM, Robson AM, Russell-Jones R, Barlow RJ: Imiquimod and lentigo maligna: a search for prognostic features in a clinicopathological study with long-term follow-up. Br J Dermatol 2009;160:994-998.

-2 Wolf IH, Kodama K, Cerroni L, Kerl H: Nature of inflammatory infiltrate in superficial cutaneous malignancies during topical imiquimod treatment. Am J Dermapathol 2007;29:237-240.

-3 Ahmed I, Berth-Jones J: Imiquimod: a novel treatment for lentigo maligna. Br J Dermatol 2000;143:843-845.

-4 Rapjar SF, Marsden JR: Imiquimod in the treatment of lentigo maligna. Br J Dermatol 2006;155:653-656.

-5 Buettiker UV, Yawalkar NY, Braathen LR, Hunger RE: Imiquimod treatment of lentigo maligna: an open-label study of 34 primary lesions in 32 patients. Arch Dermatol 2008;144:943-945.

6 Naylor MF, Crowson N, Kuwahara R, et al: Treatment of lentigo maligna with topical imiquimod. Br J Dermatol 2003;149(suppl 66):66-70.

-7 Spenny ML, Walford J, Wernchniak AE, et al: Lentigo maligna (melanoma in situ) treated with imiquimod cream 5\%: 12 case reports. Cutis 2007;79:149-152.

8 Fisher GH, Lang PG: Treatment of melanoma in situ on sun-damaged skin with topical $5 \%$ imiquimod cream complicated by the development of invasive disease. Arch Dermatol 2003;139:945-947.

-9 Fleming CJ, Bryden AM, Evans A, Dawe RS, Ibbotson SH: A pilot study of treatment of lentigo maligna with 5\% imiquimod cream. Br J Dermatol 2004;151:485-488.

10 van Meurs T, van Doorn R, Kirtschig G: Recurrence of lentigo maligna after initial complete response to treatment with 5\% imiquimod cream. Dermatol Surg 2007;33:623-627. 